\title{
A high-light sensitivity optical neural silencer: development and application to optogenetic control of non-human primate cortex
}

\author{
Xue Han ${ }^{1,2+}$, Brian Y. Chow ${ }^{1,2+}$, Huihui Zhou ${ }^{2}$, Nathan C. Klapoetke ${ }^{1,2}$, Amy Chuong $^{1,2}$, Reza Rajimehr $^{2}$, \\ Aimei Yang 1,2, Michael V. Baratta ${ }^{1,2}$, Jonathan Winkle ${ }^{2}$, Robert Desimone ${ }^{2}$ and Edward S. Boyden ${ }^{1,2 *}$ \\ Synthetic Neurobiology Group, Media Lab and Department of Biological Engineering, Massachusetts Institute of Technology, Cambridge, MA, USA \\ 2 Department of Brain and Cognitive Sciences, McGovern Institute for Brain Research, Massachusetts Institute of Technology, Cambridge, MA, USA
}

\section{Edited By:}

Andrew J. Parker, University of Oxford, UK

\section{Reviewed By:}

Andrew J. Parker, University of Oxford, UK

Hendrikje Nienborg, Salk Institute for Biological Studies, USA

\section{*Correspondence:}

Edward S. Boyden, Media Lab, Brain and Cognitive Sciences, and Biological Engineering, Massachusetts Institute of Technology, E15-473B, 20 Ames

Street, Cambridge, MA 02139, USA. e-mail:esb@media.mit.edu

${ }^{+}$Xue Han and Brian Y. Chow have contributed equally to this work.
Technologies for silencing the electrical activity of genetically targeted neurons in the brain are important for assessing the contribution of specific cell types and pathways toward behaviors and pathologies. Recently we found that archaerhodopsin-3 from Halorubrum sodomense (Arch), a light-driven outward proton pump, when genetically expressed in neurons, enables them to be powerfully, transiently, and repeatedly silenced in response to pulses of light. Because of the impressive characteristics of Arch, we explored the optogenetic utility of opsins with high sequence homology to Arch, from archaea of the Halorubrum genus. We found that the archaerhodopsin from Halorubrum strain TP009, which we named ArchT, could mediate photocurrents of similar maximum amplitude to those of Arch ( $900 \mathrm{pA}$ in vitro), but with a $>3$-fold improvement in light sensitivity over Arch, most notably in the optogenetic range of $1-10 \mathrm{~mW} / \mathrm{mm}^{2}$, equating to $>2 x$ increase in brain tissue volume addressed by a typical single optical fiber. Upon expression in mouse or rhesus macaque cortical neurons, ArchT expressed well on neuronal membranes, including excellent trafficking for long distances down neuronal axons. The high light sensitivity prompted us to explore ArchT use in the cortex of the rhesus macaque. Optical perturbation of ArchT-expressing neurons in the brain of an awake rhesus macaque resulted in a rapid and complete $(\sim 100 \%)$ silencing of most recorded cells, with suppressed cells achieving a median firing rate of 0 spikes/s upon illumination. A small population of neurons showed increased firing rates at long latencies following the onset of light stimulation, suggesting the existence of a mechanism of network-level neural activity balancing. The powerful net suppression of activity suggests that ArchT silencing technology might be of great use not only in the causal analysis of neural circuits, but may have therapeutic applications.

Keywords: channelrhodopsin, halorhodopsin, archaerhodopsin, optogenetics, non-human primate, systems neuroscience, neurophysiology, neural silencing

\section{INTRODUCTION}

Technologies for transiently silencing the activity of specific neurons in the brain are beginning to open up new frontiers on the neuroscientific investigation of normal and pathological cognition, perception, and action, and also are pointing the way for new prototype neuromodulation strategies for treating brain disorders. Since the discovery that the light-driven inward chloride pump halorhodopsin from N. pharaonis could, when expressed in neurons, mediate light-driven quieting of neural activity (Han and Boyden, 2007; Zhang et al., 2007), a number of other natural and engineered light-activated molecules have proven useful in quieting neural activity in the brain in response to light (Gradinaru et al., 2008, 2010; Chow et al., 2010; Knöpfel et al., 2010). We recently discovered that archaerhodopsin-3 from Halorubrum sodomense (Arch), a light-driven outward proton pump, could when expressed in neurons enable them to be powerfully and transiently silenced in response to pulses of light (Chow et al., 2010). Arch supported inhibitory currents approach- ing $900 \mathrm{pA}$ in vitro, enabling $\sim 100 \%$ silencing of neurons in the awake mouse brain. Importantly, we found that whereas halorhodopsins appeared to enter long-lasting inactive states (e.g., with time constants of recovery of many minutes) in response to illumination, Arch could rapidly recover its ability to be light-driven after being illuminated, important for scientific experiments in which repeated silencing is desired, such as repeated behavioral trials. Here, we explored the ability of opsins with high sequence homology to Arch to hyperpolarize neurons, and discovered that the archaerhodopsin from Halorubrum strain TP009, which we named ArchT, was $>3 \times$ more light sensitive than Arch, equating to $>2 \times$ increase in tissue volume addressed by a typical single optical fiber; the improvement was statistically significant for irradiances in the optogenetic range of $1-10 \mathrm{~mW} / \mathrm{mm}^{2}$. We also found that ArchT expressed extremely well on the membranes of neurons, including good expression on axons, which may support improved neural pathway silencing. Prompted by this enhanced light sensitivity, we demonstrated the efficacy of ArchT to mediate 
neural silencing in the cortex of an awake primate. We anticipate that ArchT will be useful for many neural silencing experiments in neuroscience, and given its demonstration of use in the nonhuman primate brain, may support new kinds of prototype treatment for neural disorders.

\section{MATERIALS AND METHODS USE OF EXPERIMENTAL ANIMALS}

All procedures were in accordance with the National Institutes of Health Guide for the care and use of Laboratory Animals and approved by the Massachusetts Institute of Technology Animal Care and Use Committee. All primate procedures were in accordance with the National Institutes of Health Guide for the care and use of Laboratory Animals and approved by the Massachusetts Institute of Technology Animal Care and Use Committee.

\section{IN VITRO METHODS}

In vitro methods were as described in Chow et al. (2010). Briefly, opsins were mammalian codon-optimized, and were synthesized by Genscript (Genscript Corp., NJ, USA). Opsins were fused in frame, without stop codons, ahead of GFP (using BamHI and AgeI) in a lentiviral vector containing the CaMKII promoter (FCK-ArchT-GFP). Codon-optimized sequences of ArchT and ArchT-GFP are stored in GENBANK (http://www.ncbi.nlm.nih. gov/) at accession numbers: HM367071, ArchT, HM367072, ArchTGFP. Genes are available for request at the link http://syntheticneurobiology.org/protocols. The amino acid sequence of ArchT is: MDPIALQAGYDLLGDGRPETLWLGIGTLLMLIGTFYFIVKG WGVTDKEAREYYSITILVPGIASAAYLSMFFGIGLTEVTVAGEV LDIYYARYADWLFTTPLLLLDLALLAKVDRVSIGTLVGVDALM IVTGLIGALSHTPLARYSWWLFSTICMIVVLYFLATSLRAAAKE RGPEVASTFNTLTALVLVLWTAYPILWIIGTEGAGVVGLGIETL LFMVLDVTAKVGFGFILLRSRAILGDTEAPEP.

Swiss Webster or C57 mice (Taconic or Jackson Labs) were used. Hippocampal and cortical cultures were prepared as described in Chow et al. (2010). Neurons were transfected at 3-5 days in vitro using calcium phosphate (Invitrogen). GFP fluorescence was used to identify successfully transfected neurons.

Whole-cell patch clamp recordings were made on neurons at 9-14 days in vitro, using a Multiclamp 700B amplifier, Digidata 1440 digitizer, and a PC running pClamp (Molecular Devices). During recording, neurons were bathed in Tyrode solution containing (in mM): $125 \mathrm{NaCl}, 2 \mathrm{KCl}, 3 \mathrm{CaCl}_{2}, 1 \mathrm{MgCl}_{2}, 10$ HEPES, 30 glucose, $0.01 \mathrm{NBQX}$, and 0.01 gabazine, at $\mathrm{pH} 7.3(\mathrm{NaOH}$ adjusted), and with 305-310 mOsm (sucrose adjusted). Borosilicate glass (Warner) pipettes were filled with a solution containing (in $\mathrm{mM}$ ): $125 \mathrm{~K}$-Gluconate, $8 \mathrm{NaCl}, 0.1 \mathrm{CaCl}_{2}, 0.6 \mathrm{MgCl}_{2}, 1 \mathrm{EGTA}$, 10 HEPES, $4 \mathrm{Mg}$-ATP, $0.4 \mathrm{Na}-\mathrm{GTP}$, at $\mathrm{pH} 7.3$ (KOH adjusted), and with 295-300 mOsm (sucrose adjusted). Pipette resistance was 5-10 M 2 ; access resistance was 10-30 M $\Omega$, monitored throughout the voltage-clamp recording; resting membrane potential was approximately $-60 \mathrm{mV}$ in current-clamp recording. To assess proton pumping by previously uncharacterized opsins, neurons were bathed in recording solution containing (in $\mathrm{mM}$ ): $125 \mathrm{~N}$-methyl-D-glucamine, $2 \mathrm{Cs}$-methanesulfonate, $3 \mathrm{CdSO}_{4}, 1$ $\mathrm{MgSO}_{4}$, 10 HEPES, 30 glucose, 0.01 NBQX, 0.01 gabazine, $\mathrm{pH} 7.3$ ( $\mathrm{H}_{2} \mathrm{SO}_{4}$ adjusted), 305-310 mOsm (sucrose adjusted), and pipettes were also filled with analogous solutions containing (in $\mathrm{mM}$ ): $125 \mathrm{Cs}$-methanesulfonate, $8 \mathrm{~N}$-methyl-D-glucamine, $0.1 \mathrm{CdSO}_{4}$, $0.6 \mathrm{MgSO}_{4}$, 1 EGTA, 10 HEPES, $4 \mathrm{Mg}-\mathrm{ATP}, 0.4$ Tris-GTP, pH 7.3 ( $\mathrm{CsOH}$ adjusted), 295-300 mOsm (sucrose adjusted).

Photocurrents were measured with 1- or 15-s duration light pulses in neurons voltage clamped at $-60 \mathrm{mV}$. Light-induced membrane hyperpolarizations were measured with 1-s light pulses, in neurons current clamped at their resting membrane potential. For all experiments except for the action spectrum characterization experiments, a DG-4 optical switch with $300 \mathrm{~W}$ xenon lamp (Sutter Instruments) was used to deliver light pulses. The DG-4 was controlled via TTL pulses generated through a Digidata signal generator. A $575 \pm 25 \mathrm{~nm}$ bandpass filter (Chroma) was used to deliver yellow light. In order to extend our power characterization of Arch and ArchT beyond the power of the yellow light available on our microscope using the configuration that we utilized, we extrapolated to higher effective yellow powers by equating various powers of unfiltered white light illumination from the DG4, to approximate effective yellow power equivalents, as done in Chow et al. (2010). For action spectrum characterization, a Till Photonics PolyChrome V, $150 \mathrm{~W}$ xenon, $15 \mathrm{~nm}$ monochromator bandwidth, was used. Data was analyzed using Clampfit (Molecular Devices) and MATLAB (Mathworks, Inc.). Statistical analysis and curve fitting was done with Statview (SAS Institute), MATLAB, GraphPad, and Origin (OriginLab).

\section{VIRUS PREPARATION}

Replication-incompetent VSVg-pseudotyped lentivirus FCKArchT-GFP was produced as previously described (Han et al., 2009; Chow et al., 2010), which allows the preparation of clean, non-toxic, high-titer virus (estimated at $\sim 10^{9}-10^{10}$ infectious units/ml). Briefly, HEK293FT cells (Invitrogen) were transfected with the lentiviral plasmid, the viral helper plasmid $\mathrm{p} \Delta 8.74$, and the pseudotyping plasmid pMD2.G. The supernatant of transfected HEK cells containing virus was then collected $48 \mathrm{~h}$ after transfection, purified, and then pelleted through ultracentrifugation. Lentivirus pellet was resuspended in phosphate buffered saline (PBS) and stored at $-80^{\circ} \mathrm{C}$.

\section{MOUSE VIRUS INJECTION AND DATA ANALYSIS}

Mouse virus injection and data analysis were as described in Chow et al. (2010). Under isoflurane anesthesia, $1 \mu$ FCK-ArchTGFP lentivirus was injected through a craniotomy made in the mouse skull, into the motor cortex (stereotaxic coordinates from Bregma, anterior-posterior: $+1.10 \mathrm{~mm}$, dorsal-ventral: $-0.5 \mathrm{~mm}$, medial-lateral: $\pm 0.5 \mathrm{~mm}$ ) or infralimbic cortex (stereotaxic coordinates from Bregma, anterior-posterior: $+1.8 \mathrm{~mm}$, dorsal-ventral: $-2.0 \mathrm{~mm}$, medial-lateral: $\pm 0.25 \mathrm{~mm}$ ). Virus was injected at a rate of $0.1 \mu \mathrm{l} / \mathrm{min}$ for a total of $10 \mathrm{~min}$ after which the injector was left in place for an additional $10 \mathrm{~min}$ to allow for viral diffusion from tip. About 4-8 weeks after virus injection, mice were perfused through the left cardiac ventricle with $\sim 20 \mathrm{ml}$ $4 \%$ paraformaldehyde in PBS ( $\mathrm{pH} 7.4$ ), and then the brain was removed and sectioned into $35 \mu \mathrm{m}$ coronal sections onto slides in a cryostat at $-21^{\circ} \mathrm{C}$. Slices were mounted with Vectashield solution (Vector Labs), and visualized with a Zeiss LSM 510 or Olympus Fluoview confocal microscope using $20 \times, 40 \times$, and $63 \times$ objective lenses. 


\section{PRIMATE VIRUS INJECTION AND GENERAL SURGICAL PROCEDURES}

Primate virus injection and general surgical procedures were as previously described (Han et al., 2009). All surgical procedures were done under veterinarian-approved anesthesia and analgesia. Specifically, one rhesus monkey (Macaca mulatta), monkey A, 12 years of age, weighing $\sim 15 \mathrm{~kg}$, was used for electrophysiology; a second rhesus monkey, monkey B, 5 years of age, weighing $\sim 5 \mathrm{~kg}$ was used for histology. Under isoflurane anesthesia, a titanium headpost and recording chambers were surgically affixed to the skull. A craniotomy was opened up over the parietal region (area 7a) for monkey A, and over the visual cortex (area V1) of monkey B. Lentivirus (FCK-ArchT-GFP) was injected at a rate of $0.1-0.2 \mu \mathrm{l} /$ min at multiple depths depending on the cortex geometry. The angle of the injection was stereotactically vertical.

\section{PRIMATE OPTICAL STIMULATION AND ELECTROPHYSIOLOGICAL RECORDING}

A 100-mW green laser (SDL-532-100T, Shanghai Dream Laser Co.) was coupled to a 200-micron-diameter optical fiber (FIBER200-UV, Ocean Optics) through a homebuilt collimator setup as described (Han et al., 2009), resulting in a fiber tip irradiance of $100-200 \mathrm{~mW} / \mathrm{mm}^{2}$ "(there was some variability in power due to variability in the bevel of the fiber tips used). The laser was controlled via TTL pulses driven by a pulse generator (DS8000, WPI Inc.) with the timing controlled by a computer through the software package, Cortex (http://www.cortex.salk.edu/). Standard tungsten electrodes of 1-2 M $\Omega$ resistance (FHC, Inc.) were guided into the brain along with a fiber attached to the electrode with the tip of the electrode at the edge of a 1-mm-diameter sphere centered at the tip of the fiber. Data amplification, filtering, and acquisition were performed with a Multichannel Acquisition Processor (MAP) system (Plexon, Inc.). Spikes sampled at $40 \mathrm{kHz}$ and filtered as $250 \mathrm{~Hz}$ to $8 \mathrm{kHz}$ were semi-automatically detected using an interactively set threshold, and the spike waveforms were offline sorted using principal component analysis (PCA; Offline Sorter, Plexon, Inc.). Spike timing was defined as the moment of threshold crossing. During the experiment, one fiber and one electrode were lowered into the brain of monkey A, awake and headfixed, and optical stimulation was performed while units were recorded. During the recording period, the monkey was awake and freely viewing, in a dimly lit room. Occasional juice rewards were delivered to insure alertness, but no tasks nor reward contingencies were scheduled in recording sessions. Although optical artifacts were observed in the low-frequency LFP channel of our recordings $(0.7-170 \mathrm{~Hz})$, as we reported before (Han et al., 2009), no artifacts were observed in the spike channel of our recordings $(250 \mathrm{~Hz}$ to $8 \mathrm{kHz}$ ), as determined by a Kolmogorov-Smirnov test comparing waveform shapes in light vs. dark $(p>0.1$ all single units recorded; exemplars in Figures 2Bi,Ei).

\section{ELECTROPHYSIOLOGICAL DATA ANALYSIS AND STATISTICS}

Electrophysiological data analysis was performed using MATLAB (Mathworks, Inc.), and all statistics were analyzed with JMP, MATLAB, and Excel. Light-modulated neurons were identified and classified by performing a paired $t$-test, for each neuron, between the firing rate during the 2 -s period before light onset vs. during the 1-s period of light exposure, across all trials for that neuron, and thresholded at the $p<0.05$ significance level. Units that showed significant decrease in firing rate during light exposure were defined as suppressed ( $n=45$ single units); units that showed significant increases in firing rate during light exposure were defined as excited ( $n=7$ single units). Twenty-two single units recorded showed no modulation by light, perhaps reflecting neurons neither ArchTpositive nor connected to ArchT-expressing neurons.

Instantaneous firing rate histograms were computed by averaging the instantaneous firing rate for each neuron across all trials recorded for that neuron, with a histogram time bin of $20 \mathrm{~ms}$. To determine the latency between light onset and the neural response to light, we performed for each neuron, and for each $20 \mathrm{~ms}$ bin during the 1-s of light exposure, a one-sample $t$-test of the instantaneous firing rates during the one hundred $20 \mathrm{~ms}$ bins in the $2 \mathrm{~s}$ before light onset, against the instantaneous firing rate during the $20 \mathrm{~ms}$ bin during light exposure under consideration. Latency was defined as the time from light onset to the time at which firing rate was significantly different from baseline $(p<0.05)$ for each of three successive $20 \mathrm{~ms}$ bins. The time for after-light recovery back to baseline was calculated similarly, testing the set of baseline bins against each of the $20 \mathrm{~ms}$ bins after light offset.

To determine peak inhibition, we swept a 200-ms-long sliding window in $1 \mathrm{~ms}$ increments through the electrophysiology data while a neuron was illuminated, and calculated the firing rate for each of these windows. (Thus, the last time window covered the period of 999-1199 ms after light onset.) Peak inhibition was defined as the ratio of lowest firing rate during light illumination period and the baseline firing rate calculated for the 2-s period before light onset. Peak increases in activity were analyzed similarly.

\section{PRIMATE HISTOLOGY}

Primate histology was performed as previously described (Han et al., 2009). Under isoflurane anesthesia, lentivirus (FCK-ArchTGFP) was injected at a rate of $0.2 \mu \mathrm{l} / \mathrm{min}$ into the visual cortex of one rhesus macaque. Perfusion was performed 1 month after virus injection, and GFP fluorescence was visualized with an Olympus FV1000 confocal microscope using a $60 \times$ objective lens.

\section{RESULTS}

We recently performed a broad screen of microbial rhodopsins (or "opsins") from species found in different kingdoms of life, characterizing the neural silencing capability of different kinds of light-driven ion pump (Han and Boyden, 2007; Chow et al., 2010). In our previous study, we found that the light-driven outward proton pump archaerhodopsin-3 (Arch), from the archaebacterium Halorubrum sodomense, was capable of mediating strong, reliable, and safe neural silencing in response to green-yellow light, likely due to its good functional expression in neurons, its fast photocycle, its rapid recovery kinetics after illumination, and the excellent proton handling capability of neurons. Because of the impressive characteristics of Arch, we explored the neural silencing capability of opsins with high sequence homology to Arch, cloned from archaeal species in the Halorubrum genus and isolated from Tibet, Australia, and other parts of the world. All electrogenic members of this class of opsins, known as archaerhodopsins, expressed well on the membranes of neurons, and demonstrated large hyperpolarizing currents when transfected into cultured hippocampal or cortical neurons and illuminated (Figure 1A). All of 
A

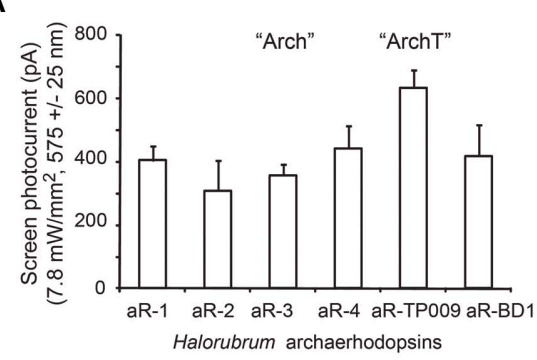

B

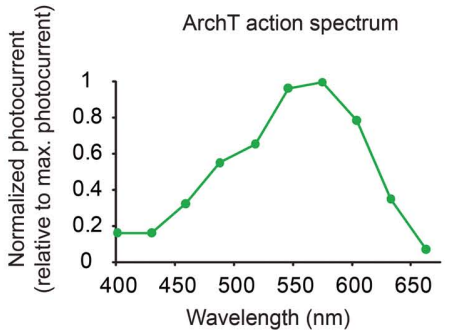

C

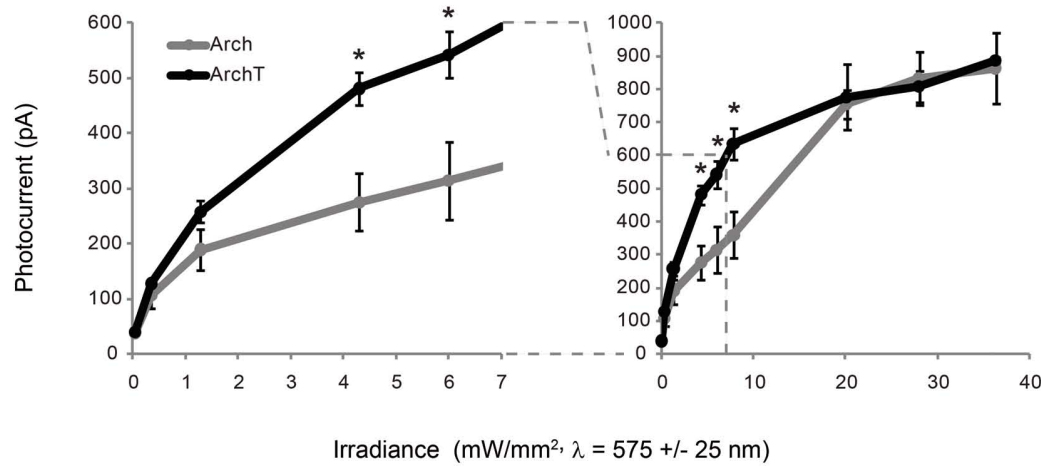

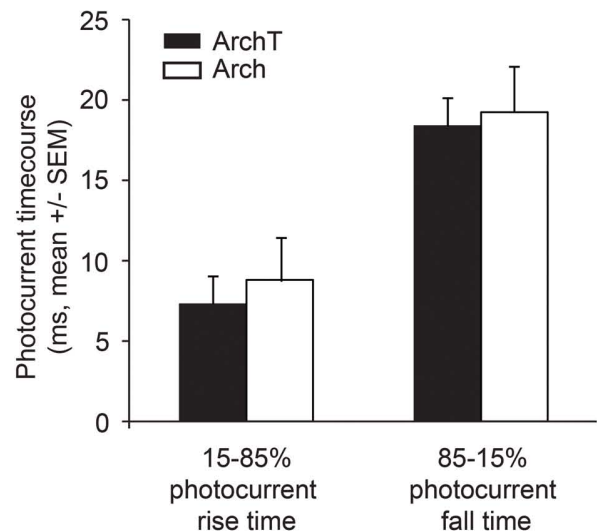

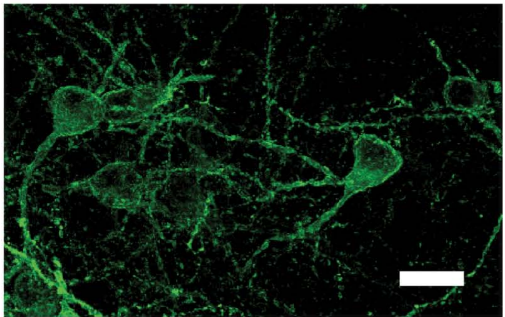

ii

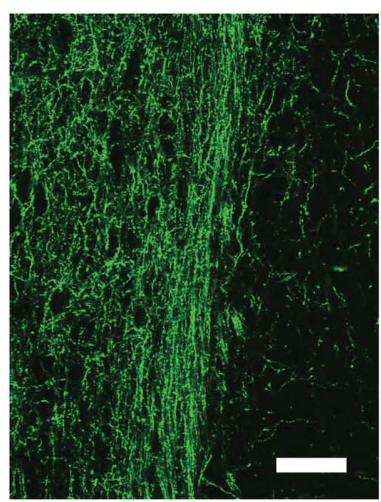

FIGURE 1|ArchT, a novel high-light sensitivity optical neural silencer. (A) Screen photocurrents generated by various opsins within the archaerhodopsin class, as assessed in neuron culture ( $n=5-16$ neurons for each bar), measured by whole-cell voltage clamp under standardized screening illumination conditions (7.8 mW/mm², $575 \pm 25 \mathrm{~nm}$ illumination). Abbreviations: aR-1, archaerhodopsin from Halorubrum strain aus-1; aR-2, archaerhodopsin from Halorubrum strain aus-2; aR-3, archaerhodopsin from Halorubrum sodomense (aka "Arch"); aR-4, archaerhodopsin from Halorubrum strain xz515; aR-TP009, archaerhodopsin from Halorubrum strain TP009 (aka "ArchT"); aR-BD1, archaerhodopsin from Halorubrum xingijanense. (B) Action spectrum of ArchT measured in cultured neurons by scanning illumination light wavelength through the visible spectrum ( $n=8$ neurons). (C) Photocurrents of ArchT and the previously published reagent Arch measured as a function of $575 \pm 25 \mathrm{~nm}$ light irradiance ( $n=4-16$ neurons for each point); curves are Hill plots. ${ }^{*} p<0.05$, $t$-test. (D) Rise times and fall times (measured from the time that the current takes to ramp up from 15 to $85 \%$ of its maximum value, and to decline by the same amount) for ArchT vs. Arch-mediated photocurrents in neurons. (Ei) Fluorescence image showing lentiviral ArchT-GFP expression in mouse motor cortex, 1 month after lentiviral injection. Scale bar, $15 \mu \mathrm{m}$. (Eii) Fluorescence image showing lentiviral ArchT-GFP expression in the axons of mouse infralimbic cortex neurons, here projecting into the nucleus accumbens shell, $\sim 1$ month after lentiviral injection. Scale bar, $45 \mu \mathrm{m}$. 
these opsins also exhibited rapid kinetics of photocurrent rise and fall, as well as good recovery of currents after illumination. Finally, these opsins exhibited similar action spectra (e.g., a representative action spectrum is shown in Figure 1B) to that of Arch, and were proton pumps as evidenced by presence of current in the absence of sodium, potassium, calcium, and chloride ions.

Particularly impressive were the hyperpolarizing currents observed upon expression of the archaerhodopsin from Halorubrum strain TP009, which we named ArchT. ArchT differs from Arch in amino acid sequence in several places within putative cytoplasmic and extracellular domains, and exhibited a 3.3-fold increase in light sensitivity over Arch (Figure 1C), with similar maximum current to Arch ( $900 \mathrm{pA}$ in vitro), and similar kinetics of photocurrent rise and fall [15-85\% photocurrent rise time of $7.4 \pm 5.5 \mathrm{~ms}$, mean $\pm \mathrm{SD} ; 85-15 \%$ fall time $18.4 \pm 9.0 \mathrm{~ms} ; n=11$ neurons; $p>0.5$ in comparison of either to that of Arch using published data from Chow et al. (2010), $t$-test; Figure 1D]. Note that because the photocurrent-irradiance curve is sigmoidal, the 3.3-fold increase in light sensitivity means that the improvements are most manifest in the range of irradiances where the photocurrents exhibit their steepest slopes, e.g., between 1 and $10 \mathrm{~mW} / \mathrm{mm}^{2}$; indeed, a point-by-point $t$-test asking whether there is improvement in photocurrent at a given irradiance shows significant ArchT improvements over Arch in this range (datapoints flagged by * in Figure 1C, indicating $p<0.05, t$-test of ArchT photocurrents vs. Arch photocurrents). We did not examine irradiances greater than $36.3 \mathrm{~mW} / \mathrm{mm}^{2}$ (the rightmost datapoint in Figure 1C), although irradiances as high as $200-500 \mathrm{~mW} / \mathrm{mm}^{2}$ are used in vivo; accordingly, we would anticipate that the maximum degree of inhibition would be similar between neurons expressing Arch and neurons expressing ArchT, but the volume that would be inhibited would be larger with ArchT. According to our earlier computational models (Chow et al., 2010), a 3× improvement in light sensitivity would increase the volume of tissue addressed by a typical single optical fiber by $>2 \times$, making ArchT of instant utility for silencing larger brain volumes. Voltage swings in current clamped hippocampal neurons were similarly fast (15-85\% voltage decline time of $15.0 \pm 3.9 \mathrm{~ms}$, mean $\pm \mathrm{SD}$; $85-15 \%$ recovery time $45.2 \pm 34.3 \mathrm{~ms}$; $n=9$ neurons). Thus, ArchT could mediate fast hyperpolarization of neurons, with excellent light sensitivity. When we genetically targeted ArchT to cortical pyramidal neurons of the mouse brain under the CaMKII promoter using a lentivirus (Han et al., 2009), we observed good expression, as well as good trafficking to the cell surface (Figure 1Ei). In addition, we observed excellent and rapid expression along the length of axonal projection pathways traveling far from the cell body (Figure 1Eii), suggesting utility in silencing defined projection pathways between different regions in the brain.

Prompted by the high-light sensitivity of ArchT, we used the aforementioned lentivirus to genetically target ArchT to cortical pyramidal neurons of the cortex of the rhesus macaque. As evidenced by histological examination of ArchT-expressing neurons in cortical tissue from one monkey (monkey B), neurons appeared healthy, and expressed ArchT well, at a timepoint 1 month after viral injection (Figure 2J). When cortical neurons in a second monkey (monkey A) were recorded during illumination with a fiber coupled to an inexpensive $532 \mathrm{~nm}$ laser (fiber tip irradiance $100-200 \mathrm{~mW} / \mathrm{mm}^{2}$, electrode tip at edge of $\sim 1 \mathrm{~mm}$-diameter sphere centered at fiber tip (Chow et al., 2010), 1-3 months after viral injection (see Figure 2A for experimental timeline), clear silencing of activity by $\sim 100 \%$ was observed in the majority of recorded cells, in response to light (Figures $\mathbf{2 B}, \mathbf{C}, \mathbf{H}$ ), with a median resultant firing rate of 0 spikes/s (i.e., total silencing) among the neurons which showed any decrease in firing upon light delivery (Figure 2D; range, $81.3-100.0 \%$ peak percent reductions in firing rate; $n=45$ silenced single units, out of 74 total single units recorded). Thus, ArchT safely expressed over periods of many months, and could support high-light sensitivity optical quieting of neural network activity, in the cortex of an awake macaque. The non-Gaussian appearance of the distribution of silencing efficacies may be due to the fact that some neurons that decreased activity during illumination were not ArchT-positive, but instead decreased their activity because they were downstream of cells that were directly silenced by illumination.

To explore the properties of neural population silencing further, we analyzed the temporal dynamics of the neural silencing. For the silenced single units, decreases in spike activity began rapidly after light onset, with a median latency of $60 \mathrm{~ms}$ (Figure 2G; range, 0-400 ms). After light cessation, activity in silenced cells recovered to baseline firing rate after a median of $740 \mathrm{~ms}$ (range, 0-4260 ms), without showing sharp rebound activity.

Interestingly, 7 of the 74 recorded neurons did not show reductions in firing rate, but instead underwent significant increases in spiking activity during light delivery to ArchT-expressing pyramidal cells. These neurons increased their firing rate by a median of 2.9-fold (range, 2.0- to 10.6-fold; Figures 2E,F,I). These neurons began increasing spiking with a latency of $280 \mathrm{~ms}$ after light onset (median; range, 20-740 ms), significantly longer than the latency for initiating optical quieting of the primary population of light-silenced neurons ( $p<0.01$, Mann-Whitney $U$-test). These increased-activity neurons were recorded at locations that were interspersed amongst the locations where silenced neurons were recorded.

We used spike waveforms shapes to analyze the cell-type identity of the silenced and increased-activity neurons. Six of the 45 silenced neurons had narrow spike waveforms with trough-to-peak durations of 100-200 $\mu$ s, suggestive of putative inhibitory neurons, whereas the remainder had broader spike waveforms, suggestive of putative excitatory neurons (Mitchell et al., 2007). Three of the seven increased-activity neurons had narrow spike waveforms, suggestive of inhibitory neurons (Mitchell et al., 2007). Thus, it is likely that both the silenced and increased-activity populations of neurons comprised mixed populations of pyramidal cells and inhibitory cells. Baseline firing rates of the two populations of neurons were not different ( $p>0.09$, Mann-Whitney $U$-test).

\section{DISCUSSION}

We here present a novel optical neural silencing tool that expresses well on the membranes of neurons, including good expression along axons of neurons, and operates well in the context of the awake mammalian brain. Like our earlier-characterized reagent Arch (Chow et al., 2010), ArchT supports light-driven quieting of neural activity with high efficacy and fast kinetics, utilizing inexpensive $532 \mathrm{~nm}$ lasers (typically, an order of magnitude cheaper than yellow lasers) for illumination. The key difference is that ArchT has 


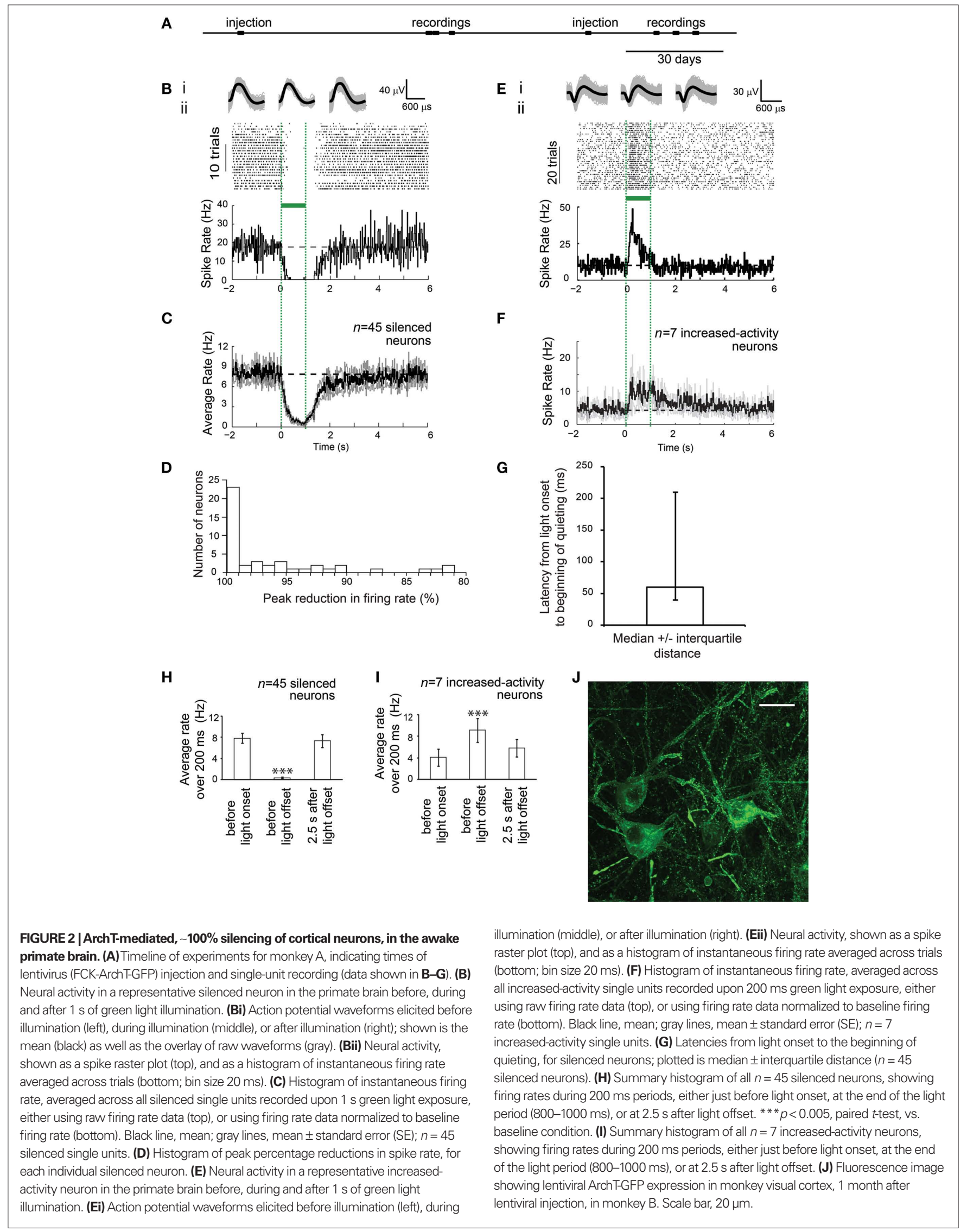


$>3 \times$ better light sensitivity over Arch (with statistically significant improvements in photocurrent of ArchT over Arch, when light $1-10 \mathrm{~mW} / \mathrm{mm}^{2}$ is delivered), increasing by $2 \times$ the volume of tissue addressed by a typical optical fiber.

Using ArchT delivered by lentivirus, a strategy that selects for excitatory cells being labeled, the majority of neurons in the awake primate recorded exhibited $\sim 100 \%$ silencing when illuminated, achieving a median firing rate of 0 spikes/s. Thus, ArchT may be useful for neuroscientific investigations of the neural mechanisms of behavior, especially when large brain regions are required to be silenced, or when animals such as macaques are under consideration. The class of archaerhodopsins may present structural features that enable particularly good expression in mammalian cells, and may serve in the future as a template for the generation of further molecular tools that subserve effective optical control of biological functions in basic science and therapeutic contexts.

We previously showed (Han et al., 2009) that brief optical excitation of a population of pyramidal cells (expressing the depolarizing light-gated cation channel channelrhodopsin-2 (ChR2; Nagel et al., 2003; Boyden et al., 2005) resulted in a large population of excited cells and a significant minority of suppressed cells. This early study, taken together with the current study, suggests a novel principle governing how the cortex reacts to control of the activity of specific cell populations. Specifically, our results suggest that when a population of cells in the cortex is directly perturbed either via excitation or silencing, a secondary response emerges in another population of cells, which in part balances (e.g., homeostatically) network activity by opposing the primary response driven by direct control. The secondary opponent response arises with a longer latency than the primary response. This finding is suggestive of the possibility that specific circuit elements, such as inhibitory interneurons, are playing a role in the balancing of total cortical neural activity upon pyramidal cell activity perturbation. That is, the silencing of pyramidal neurons decreases the drive to inhibitory neurons, thus increasing activity in cortical cells targeted by those inhibitory neurons; conversely, exciting pyramidal neurons increases the drive to inhibitory neurons, decreasing activity in downstream cortical neurons. Of course, these secondary responses may also modulate the primary responses. For example, a neuron that increases its activity as a secondary response to pyramidal cell silencing, if it synapses upon a neuron undergoing a primary silencing response, may lessen or slow this primary silencing response. Such principles, which predict how a neural circuit will react to a neural manipulation, will be increasingly important if neural control tools are to be used in algorithmic fashion to dictate the state of a brain network.

\section{REFERENCES}

Bainbridge, J. W., Smith, A. J., Barker, S. S., Robbie, S., Henderson, R., Balaggan, K., Viswanathan, A., Holder, G. E., Stockman, A., Tyler, N., PetersenJones, S., Bhattacharya, S. S., Thrasher, A. J., Fitzke, F. W., Carter, B. J., Rubin, G. S., Moore, A. T., and Ali, R. R. (2008). Effect of gene therapy on visual function in Leber's congenital amaurosis. N. Engl. J. Med. 358, 2231-2239.
Nature Biotechnology, Editorial (2007). 949.

Boyden, E. S., Zhang, F., Bamberg, E., Nagel, G., and Deisseroth, K. (2005). Millisecond-timescale, genetically targeted optical control of neural activity. Nat. Neurosci. 8, 1263-1268.

Chow, B. Y., Han, X., Dobry, A. S., Qian, X., Chuong, A. S., Li, M., Henninger, M.A., Belfort, G. M., Lin, Y., Monahan, P. E., and Boyden, E. S. (2010). HighRetracing events. Nat. Biotechnol. 25,

It is important to point out that other explanations are possible, e.g., cortical silencing could have unmasked other projections into cortex that result in excitation, or silencing of neurons in cortex could have resulted in increased-activity neurons through other indirect means.

Although only two monkeys were here studied (one monkey was considered for histological analysis, and one monkey considered for optical/electrophysiological analysis), our work points to the possibility that novel therapies based upon cell-type specific neural silencing may be of use in the future. Already neural stimulation strategies such as deep brain stimulation (DBS), in which temporally precise electrical stimulation is applied to neural targets via a chronically implanted electrode, are used in tens of thousands of patients for the treatment of Parkinson's disease, depression, obsessive-compulsive disorder, Tourette's, and other disorders (Perlmutter and Mink, 2006). A technology for the safe, temporally precise, direct silencing of neurons could open up a new front in the neuromodulatory treatment of brain disorders, enabling the suppression of dysfunctional cell populations. Given that gene therapy strategies are beginning to achieve enduring and safe expression of genes in neurons in human patients (Biotechnol, 2007; Kaplitt et al., 2007; Bainbridge et al., 2008), it is possible that molecules like ArchT, if proven safe by future experiments, may be useful for dynamic physiological control, beyond what has been enabled by gene therapies used in a translational setting to date.

\section{ACKNOWLEDGMENTS}

We thank members of the Boyden and Desimone labs for helpful discussion. We thank the Center for Bits and Atoms and its director Neil Gershenfeld for use of its facilities. This work was supported by a HHWF Fellowship and NIH K99MH085944 to Xue Han; a McGovern Institute Neurotechnology (MINT) Award to Edward S. Boyden, Robert Desimone, and Xue Han; grants NIH-EY017292 and EY017921 to Robert Desimone; and grant NIH 1RC1MH088182 to Robert Desimone and Edward S. Boyden. Edward S. Boyden acknowledges support from the NIH (Director's New Innovator Award 1DP2OD002002, 1R01DA029639, 1R43NS070453, 1RC2DE020919, 1R01NS067199), NSF (EFRI 0835878, DMS 0848804), Department of Defense, NARSAD, Alfred P. Sloan Foundation, Jerry and Marge Burnett, SFN Research Award for Innovation in Neuroscience, MIT Media Lab, MIT McGovern Institute, Benesse Foundation, MIT Neurotechnology Fund, and Wallace H. Coulter Foundation. The funders had no role in study design, data collection and analysis, decision to publish, or preparation of the manuscript.

performance genetically targetable optical neural silencing by light-driven proton pumps. Nature 463, 98-102.

Gradinaru, V., Thompson, K. R., and Deisseroth, K. (2008). eNpHR: a Natronomonas halorhodopsin enhanced for optogenetic applications. Brain Cell Biol. 36, 129-139.

Gradinaru, V.,Zhang, F., Ramakrishnan,C. Mattis, J., Prakash, R., Diester,I., Goshen, I., Thompson, K. R., and Deisseroth, K. (2010). Molecular and cellular approaches for diversifying and extending optogenetics. Cell 141, 154-165.

Han, X., and Boyden, E. S. (2007). Multiple-color optical activation, silencing, and desynchronization of neural activity, with single-spike temporal resolution. PLoS ONE 2, e299. doi: 10.1371/journal.pone.0000299

Han, X., Qian, X., Bernstein, J. G., Zhou, H. H., Franzesi, G. T., Stern, P., Bronson, R. T., Graybiel, A. M., Desimone, R., and Boyden, E. S. (2009). Millisecond- 
timescale optical control of neural dynamics in the nonhuman primate brain. Neuron 62, 191-198.

Kaplitt,M.G., Feigin,A., Tang, C., Fitzsimons, H. L., Mattis, P., Lawlor, P.A., Bland, R. J., Young,D.,Strybing, K., Eidelberg, D., and During,M. J.(2007).Safety and tolerability of gene therapy with an adeno-associated virus (AAV) borne GAD gene for Parkinson's disease: an open label, phase I trial. Lancet 369, 2097-2105.

Knöpfel, T., Lin, M. Z., Levskaya, A., Tian, L., Lin, J. Y., and Boyden, E. S. (2010). Toward the second generation of optogenetic tools. J. Neurosci. 30, 14998-15004.

Mitchell, J. F., Sundberg, K. A., and Reynolds, J. H. (2007). Differential attention-dependent response modulation across cell classes in macaque visual area V4. Neuron 55 , 131-141.

Nagel, G., Szellas, T., Huhn, W., Kateriya, S., Adeishvili, N., Berthold, P., Ollig, D., Hegemann, P., and Bamberg, E. (2003). Channelrhodopsin-2, a directly light-gated cation-selective membrane channel. Proc. Natl. Acad. Sci. U.S.A. 100, 13940-13945.

Perlmutter, J. S., and Mink, J. W. (2006). Deep brain stimulation. Annu. Rev. Neurosci. 29, 229-257.

Zhang, F., Wang, L. P., Brauner, M., Liewald, J. F., Kay, K., Watzke, N., Wood, P. G., Bamberg, E., Nagel,
G., Gottschalk, A., and Deisseroth, K. (2007). Multimodal fast optical interrogation of neural circuitry. Nature 446, 633-639.

Conflict of Interest Statement: The authors declare that the research was conducted in the absence of any commercial or financial relationships that could be construed as a potential conflict of interest.

Received: 28 October 2010; paper pending published: 28 December 2010; accepted: 25 March 2011; published online: 13 April 2011. Citation: Han X, Chow BY, Zhou H, Klapoetke NC, Chuong A, Rajimehr R,
Yang A, Baratta MV, Winkle J, Desimone $R$ and Boyden ES (2011) A high-light sensitivity optical neural silencer: development and application to optogenetic control of non-human primate cortex. Front. Syst. Neurosci. 5:18. doi: 10.3389/ fnsys.2011.00018

Copyright (C) 2011 Han, Chow, Zhou, Klapoetke, Chuong, Rajimehr, Yang, Baratta, Winkle, Desimone and Boyden. This is an open-access article subject to a non-exclusive license between the authors and Frontiers Media SA, which permits use, distribution and reproduction in other forums, provided the original authors and source are credited and other Frontiers conditions are complied with. 